\title{
NKT-like cells reveal higher than T lymphocytes expression of cellular protective proteins HSP70 and SOD2 and comparably increased expression of SIRT1 in the oldest seniors
}

\author{
Lucyna Kaszubowska', Jerzy Foerster ${ }^{2}$, Przemyslaw Kwiatkowski ${ }^{3}$, Daria Schetz ${ }^{4}$ \\ ${ }^{1}$ Department of Histology, Medical University of Gdansk, Gdansk, Poland \\ ${ }^{2}$ Department of Social and Clinical Gerontology, Medical University of Gdansk, Gdansk, Poland \\ ${ }^{3}$ Department of Human Histology and Embryology, School of Medicine, University of Warmia and \\ Masuria in Olsztyn, Olsztyn, Poland \\ ${ }^{4}$ Department of Pharmacology, Medical University of Gdansk, Gdansk, Poland
}

\begin{abstract}
Introduction. NKT-like cells are "non-classical”, "CD1d-independent” NKT cells which represent highly differentiated, conventional T lymphocytes coexpressing several NK (natural killer) associated receptors. They are effector lymphocytes of both innate and adaptive immunity and simultaneously regulatory cells of the adaptive immune system. They reveal large granular lymphocyte morphology and can mediate both MHC-restricted and MHC-unrestricted cytotoxicity, secrete many cytokines and modulate Th1 immune responses. The aim of our study was to analyze the expression of proteins involved in cellular stress response: sirtuin 1 (SIRT1), heat shock protein 70 (HSP70) and manganese superoxide dismutase (SOD2) in NKT-like cells compared to T lymphocytes during ageing.

Material and methods. The study involved three groups of participants: the oldest seniors $(\mathrm{n}=25$; aged over 85 ; mean age $88 \pm 0.5 \mathrm{ys})$, the old $(\mathrm{n}=30$; aged under 85 ; mean age $76 \pm 0.9 \mathrm{ys})$ and the young $(\mathrm{n}=32$; mean age $21 \pm 0.3 \mathrm{ys})$. Whole blood samples were analyzed by flow cytometry to assess NKT-like $(\mathrm{CD} 3+\mathrm{CD} 56+)$ and $\mathrm{T}(\mathrm{CD} 3+)$ cell populations.

Results. The group of the oldest seniors differed from the other age groups by much higher percentage of both NKT-like cells and T lymphocytes expressing SIRT1, HSP70 and SOD2. The expression of these proteins correlated positively with the age of the participants. Interestingly, the significantly higher expression of the studied protective proteins; i.e. HSP70 and SOD2 was found in CD3+CD56 + cells compared to CD3+ lymphocytes and this phenomenon concerned all the studied age groups. These differences were not significant regarding the expression of SIRT1; however, the same tendency was noticeable.

Conclusions. The analysis of CD3+ and CD3+CD56+ lymphocytes showed the increase in the number of NKT-like cells and decreased number of T cells in the process of ageing. The increased expression of cellular protective proteins SIRT1, HSP70 and SOD2 in NKT-like and T-lymphocytes of the oldest seniors seems to correspond to longevity and the observed correlations may suggest the involvement of these proteins in establishing cellular homeostasis specific for healthy ageing. Furthermore, the higher expression of the protective proteins in NKT-like cells compared to T lymphocytes may indicate their particular role in the interplay between innate and adaptive immunity responses during the process of ageing. (Folia Histochemica et Cytobiologica 2018, Vol. 56, No. 4, 231-240)
\end{abstract}

Key words: T lymphocytes; CD3 + cells; NKT-like cells; CD3+CD56 + cells; ageing; immunosenescence; seniors; SIRT1; HSP70; SOD2 


\section{Introduction}

NKT-like cells are "non-classical”, "CD1d-independent" natural killer $\mathrm{T}$ cells which represent highly differentiated, conventional T cells coexpressing several NK-associated receptors (NKRs) such as CD56, CD161, CD16, CD94, CD57 [1-3]. They refer to the original definition of Natural Killer T (NKT) cells. Then the term "NKT cell" has been applied to name subsets of T lymphocytes expressing a semi-invariant TCR (V $\alpha 24 \mathrm{~J} \alpha 18)$ which are "classical", or "invariant" NKT cells (iNKT cells) responding to CD1d-restricted lipid ligands [4, 5]. Both subsets are differentially affected by ageing since the frequency of peripheral blood iNKT cells decreases in the elderly on the contrary to NKT-like cells which number increases with age [5-7]. Thus, the proper differentiation between these "invariant NKT" (iNKT) and "NKT-like" cells which are subsets of $\mathrm{T}$ cells is crucial particularly in biogerontology studies $[4,8]$.

NKT-like cells constitute approximately $5-15 \%$ of the peripheral T-cell population and up to $50 \%$ of $\mathrm{T}$ lymphocytes in the human liver, although the liver population is on average around 30\% [9]. They can mediate MHC-restricted and MHC-unrestricted cytotoxicity and secrete many cytokines, including IFN- $\gamma$, which induces antiviral reaction and modulates Th1 immune responses $[8,10]$. These cells show also characteristic features of NK cells, i.e. large granular lymphocyte morphology, the capacity to lyse NK cell sensitive target cell line (K562) and expression of KIR receptors and CD94 molecules after activation $[10,11]$. NKT-like cells are then both effectors of innate and adaptive immunity and potential regulators of adaptive immune response [12].

Ageing is associated with the up-regulation of the inflammatory responses caused by chronic antigenic stress and dysregulation of cytokine secretion, increased serum levels of pro-inflammatory cytokines, e.g. IL-1, IL-6 and TNF, increased level of C reactive protein and soluble cytokine receptors [13-17]. The ageing process is also accompanied by chronic oxidative stress which can cause serious damage of cells, counteracted by the endogenous defense system consisted of enzymatic antioxidants, i.e. thiol peroxidases, superoxide dismutases, catalase and non-enzymatic antioxidants, e.g. glutathione (GSH) [18-21]. Continuous low-dose oxidative stress during ageing results in adaptive responses based on the activation of NF- $\kappa \mathrm{B}$ and subsequently superoxide dismutases (SODs) with a key role played by the mitochondrial manganese superoxide dismutase (SOD2) [22, 23]. In this process also heat-shock proteins (e.g. HSP70) are induced to counteract both oxidative damage and inflammatory stress by refolding or targeting for degradation misfolded proteins. They increase the expression and activity of intracellular scavengers of reactive oxygen intermediates, i.e. catalase, superoxide dismutases and block the activation of the transcription factor NF$\kappa \mathrm{B}$, a key player in the initiation of the inflammatory response [24].

Sirtuins are evolutionary conserved proteins with deacetylase activity dependent on nicotinamide adenine nucleotide (NAD+), considered as histone deacetylases involved in the control of ageing and longevity, DNA repair, transcriptional silencing, apoptosis and cellular metabolism [25]. In mammalian cells SIRT1 directly regulates the activity of stress response transcription factors, e.g. the p53 tumor suppressor factor [26, 27], forkhead box $\mathrm{O}$ transcription factors (FOXO) $[28,29]$ and NK- $\kappa \mathrm{B}[30,31]$. SIRT1 activates several members of the FOXO family of transcription factors promoting the expression of some stress response genes including $S O D 2$ which contributes to the reduction of the level of reactive oxygen species in the cell [28, 29, 32, 33].

Despite the established role of HSP70, SIRT1 and SOD2 in immunosenescence, little is known about their contribution to ageing of NKT-like cells, a population of $\mathrm{T}$ lymphocytes, which number increases in the elderly, similarly to NK cells [5]. Likewise, there are only single studies concerning changes in the levels of these proteins in T cells in the process of ageing regarding HSP70 expression in the whole population of lymphocytes [34] and some PBL (peripheral blood lymphocyte) subsets [35] or some studies on SIRT1 expression performed on mice [36]. Therefore, the aim of our study was to analyze the expression of these cellular protective proteins in NKT-like lymphocytes $(\mathrm{CD} 3+\mathrm{CD} 56+$ cells $)$ in comparison to $\mathrm{T}$ lymphocytes (CD3 + cells) of the oldest (seniors over 85 years old), the old (seniors aged under 85 ) and the young subjects.

\section{Material and methods}

Participants. Eighty-seven volunteers aged between 19 and 94 years (63 women and 24 men) participated in this study. The exclusion criteria included: CRP $>5 \mathrm{mg} / \mathrm{L}$, cancer, autoimmune disease, diabetes, infection, use of immunosuppressors, glucocorticoids or non-steroid anti-inflammatory drugs (NSAIDs); moderate to severe dementia assessed using the "Mini Mental State Examination" (MMSE below 23 points) [37]. Senior volunteers were also considered with geriatric conditions. Katz's scale was used to assess "Activities of Daily Living" (ADL) and only seniors with 5-6 points were enrolled to the study [38]. Senior volunteers were recruited among inhabitants of local senior houses 
and young volunteers were students of Medical University of Gdansk, Poland. The participants were subdivided into 3 groups including: 32 young subjects referred to as 'young' (mean age $21.0 \pm 0.3$ years, range 19-24 years, 23 women and 9 men); 30 seniors aged under 85 referred to as 'old' (mean age $75.6 \pm 0.9$ years, range $65-84$ years, 20 women and 10 men) and 25 seniors at the age over 85 referred to as the 'oldest' (mean age $88.4 \pm 0.5$ years, range $85-94$ years; 20 women and 5 men). All volunteers signed informed consent and study received approval from Ethical Committee of the Medical University of Gdansk, Poland (225/2010). An immunological characteristics of the study population was described earlier [20].

Staining of surface and intracellular antigens for flow cytometry. Whole blood samples $(0.1 \mathrm{ml})$ were aliquoted into flow cytometry tubes and appropriate volumes of CD3-FITC-conjugated (clone UCHT1) (BD Biosciences, San Jose, CA, USA) or CD3-PE-Cy7-conjugated (clone SK7) (BD Biosciences, San Jose, CA, USA), CD56-APC-conjugated (clone NCAM16.2) (BD Biosciences, San Jose, CA, USA) and Hsp70-PE-conjugated (clone N27F34) (Abcam, Cambridge, England) monoclonal antibodies were added for cell surface antigen staining. After 30 min of incubation in the dark at room temperature $2 \mathrm{ml}$ of BD FACS Lysing Solution was added and samples were incubated for subsequent $10 \mathrm{~min}$ in the same conditions. Then cells were washed twice with $1 \mathrm{ml}$ of BD Staining Buffer (PBS without $\mathrm{Ca}^{2+}$ and $\mathrm{Mg}^{2+}, 1 \% \mathrm{FBS}, 0.09 \%$ sodium azide) and resuspended in $0.25 \mathrm{ml}$ of Fixation/Permeabilization Solution for $20 \mathrm{~min}$ at $4^{\circ} \mathrm{C}$ according to manufacturer's protocol (BD Cytofix/ /Cytoperm Fixation/Permeabilization Kit). Cells were washed twice with $1 \mathrm{ml}$ of BD Perm/Wash buffer and appropriate volumes of MnSOD-FITC-conjugated (clone MnS-1) (eBioscience, San Diego, CA, USA), Hsp70-PE-conjugated (clone N27F34) (Abcam, Cambridge, England) and SIRT1-Alexa Fluor 488 - conjugated (clone 19A7AB4) (Abcam, Cambridge, England) monoclonal antibodies were added for staining of intracellular antigens. After 30 min of incubation in the dark at room temperature cells were washed twice with $1 \mathrm{ml}$ of BD Perm/Wash buffer and resuspeded in Staining Buffer prior to flow cytometric analysis. Samples were run on a BD FACSCalibur flow cytometer equipped with argon-ion laser $(488 \mathrm{~nm})$ and data were analyzed with BD CellQuest Pro software (BD Biosciences, San Jose, CA, USA) after collecting 10,000 gated events (lymphocytes). Peripheral blood lymphocytes were gated using forward (FSC) and side scatter (SSC) parameters. T lymphocytes were identified as CD3+ cells and NKT-like cells as CD3+CD56+ cells. Relevant isotype controls for both surface and intracellular staining were also prepared. Results were then presented in two ways, i.e. as percentages of $\mathrm{CD} 3+$ and $\mathrm{CD} 3+\mathrm{CD} 56+$ cells with the expression of the studied protein (\% of positive cells) and mean fluorescence intensity (MFI).
Statistics. All data are expressed as means \pm SEM. Normality of data distribution was analyzed by Shapiro-Wilk test. ANOVA test for normal distribution and Kruskal-Wallis test for non-parametric distribution were used to compare experimental data. The multiple comparisons were performed with Tukey's post-hoc test for normal distribution and Dunn's post-hoc test for non-parametric distribution. Student's t test for normal distribution and Mann-Whitney $\mathrm{U}$ test for non-parametric distribution were used to compare two independent samples. The Spearman correlation coefficient $(\mathrm{R})$ was applied to present the strength of the relationship between variables (Statistica, version 12; Statsoft, Tulsa, OK, USA). Differences or correlations with $\mathrm{p}<0.05$ were considered as statistically significant.

\section{Results}

\section{Immunological characteristics of the study population}

As have been mentioned in Material and methods section, the studied population was divided into 3 age groups: young, old and the oldest (mean age 21, 76 and 88 years, respectively). Blood morphology of all participants was analyzed prior to the study. The numbers of CD3+ and CD3+CD56+ lymphocytes presented in $\mathrm{k} / \mu \mathrm{l}$ units and percentages of $\mathrm{CD} 3+$ and CD3 + CD56+ cells within the population of lymphocytes are shown in Table 1 . The young had more Tlymphocytes in one microliter of peripheral blood and demonstrated higher percentage of CD3 + cells in the population of lymphocytes than the elderly, although these differences were statistically significant only between young and the oldest. All compared groups, however, did not differ significantly in the number of NKT-like lymphocytes, although the old revealed the highest percentage of these cells, showing statistical significance between the old and the oldest (Table 1).

\section{Expression of SIRT1, HSP70 and SOD2 in $\mathrm{CD3}+$ and $\mathrm{CD3}+\mathrm{CD56}+$ cells of the young, old and the oldest}

The gating strategy performed for flow cytometric analysis is shown in Figures 1 and 2. Flow cytometry data were analyzed and presented in two ways, i.e. as percentages of CD3 + and CD3+CD56+ cells with the expression of the studied protein (\% of positive cells) and relative expression, i.e. mean fluorescence intensity (MFI) measured in the analyzed samples.

The expression of SIRT1 was many times higher in CD3 + cells of the oldest seniors $(6.88 \pm 1.86 \%)$ compared to the old and the young $(0.33 \pm 0.22 \%$ or $0.02 \pm 0.01 \%$, respectively) (Fig. 3A). The MFI analysis confirmed this significant increase in SIRT1 expression in T lymphocytes of the oldest seniors com- 
Table 1. Selected hematological parameters of peripheral blood in the studied age groups

\begin{tabular}{|c|c|c|c|c|}
\hline Parameters|studied group & Young & Old (seniors < 85) & Oldest (seniors $>85$ ) & $P$ value \\
\hline Lymphocyte CD3+ count $[\mathrm{k} / \mu \mathrm{l}]$ & $1.43 \pm 0.1^{*}$ & $1.23 \pm 0.08$ & $1.06 \pm 0.11^{*}$ & 0.047 \\
\hline Lymphocyte CD3+ percentage (\%) & $59.8 \pm 2.67^{*}$ & $57.58 \pm 1.59$ & $56.15 \pm 4.33^{*}$ & 0.013 \\
\hline Lymphocyte CD3+CD56+ count $[\mathrm{k} / \mu \mathrm{l}]$ & $0.12 \pm 0.01$ & $0.14 \pm 0.01$ & $0.12 \pm 0.02$ & $\mathrm{~ns}$ \\
\hline Lymphocyte CD3+CD56+ percentage (\%) & $5.12 \pm 0.44$ & $7.32 \pm 0.71^{\#}$ & $5.25 \pm 1.09^{\#}$ & 0.032 \\
\hline
\end{tabular}

All data are presented as means \pm SEM. Statistically significant differences between age groups are marked with the same upper index symbols: *young vs. oldest; \# old vs. oldest; 'ns' denotes statistically not significant. The other blood parameters of the studied groups were reported in earlier studies [20].

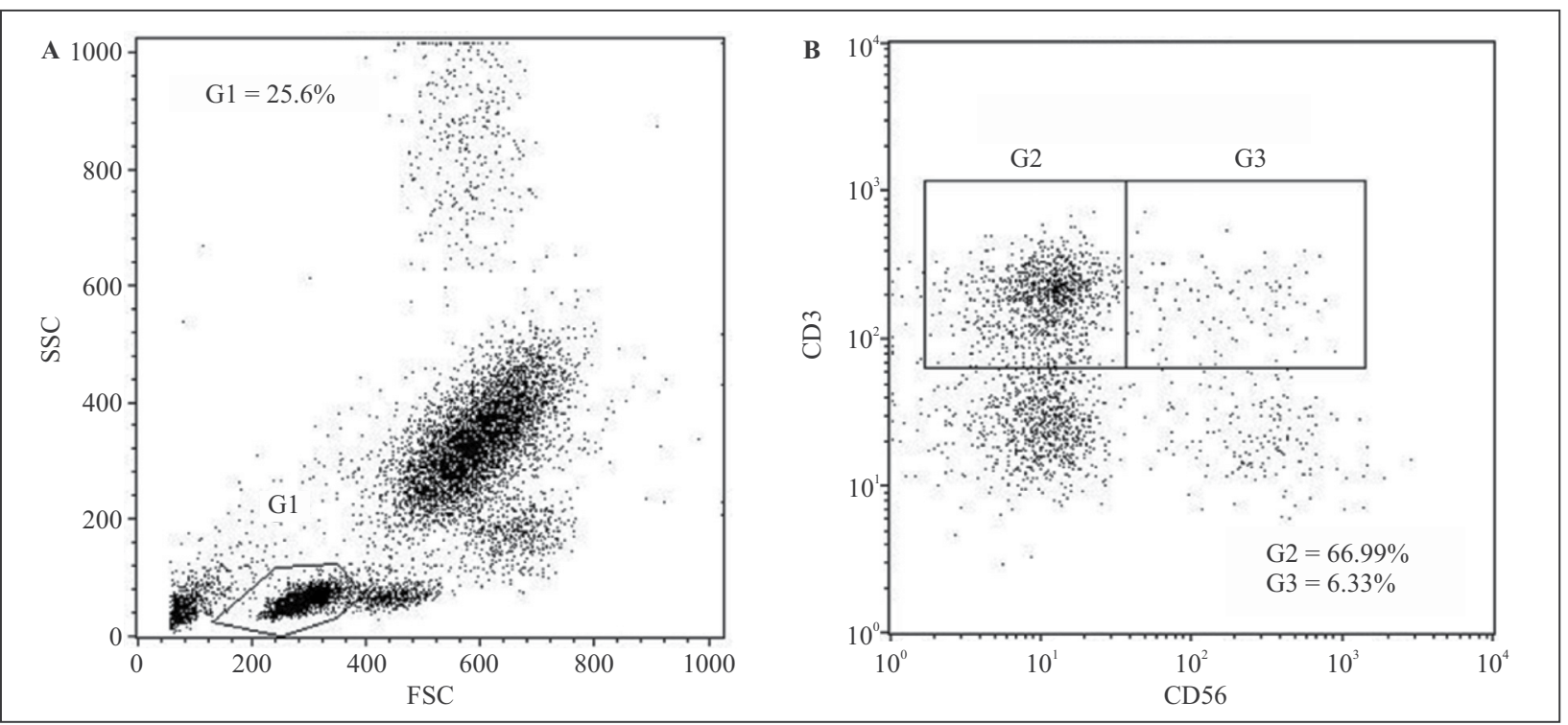

Figure 1. Gating strategy performed for flow cytometric analysis of T $(\mathrm{CD} 3+)$ and NKT-like (CD3+CD56+) lymphocytes. A. Lymphocyte gating — lymphocytes were defined as FSC ${ }^{\text {low }} / \mathrm{SSC}^{\text {low }}$ cells (G1). B. T and NKT-like cell gating - T lymphocytes were defined as CD3+ positive population (G2) and NKT-like cells as CD3+CD56+ positive cells (G3).

pared to the old and the young (Fig. 3B). Similarly, $\mathrm{CD} 3+\mathrm{CD} 56+$ cells of the oldest seniors revealed many times higher expression of SIRT1 $(10.03 \pm$ $3.09)$ in comparison to the old $(0.68 \pm 0.43)$ and the young $(0.22 \pm 0.08)$ confirmed also by the analysis of the relative expression (Fig. 3A, B). Interestingly, higher expression of SIRT1 was observed in both populations of lymphocytes in the young compared with the old when MFI analysis was performed, not observed in the analysis of percentages. There were also no differences between SIRT1 expression in $\mathrm{CD} 3+$ and $\mathrm{CD} 3+\mathrm{CD} 56+$ cells studied within the same age groups (Fig. 3A, B).

The expression of HSP70 in CD3+ cells of the oldest $(71.73 \pm 5.71 \%)$ was significantly higher than in CD3 + cells of the old and the young (29.69 \pm $\pm 4.71 \%$ and $42.90 \pm 4.2 \%$ respectively) (Fig. 3C). The expression of this cellular protective protein was even higher in NKT-like cells of the oldest $(81.97 \pm$ $\pm 4.62 \%)$ compared to the old $(42.99 \pm 4.96 \%)$ and the young (54.43 \pm 3.93 ) (Fig. 3C). The performed MFI analysis confirmed these differences between the studied groups (Fig. 3D). There were no statistically significant differences between HSP70 expression in the analyzed cells of the young and the old. Interestingly, NKT-like cells appeared to express higher level of HSP70 in comparison to T lymphocytes when these cells were compared within the same age groups shown in the analysis of the relative expression and partly in the analysis of the percentages of positive cells (Fig. 3C, D).

The expression of SOD2 in T lymphocytes of the oldest seniors $(49.50 \pm 8.09 \%)$ differed significantly from $\mathrm{T}$ lymphocytes of the old $(15.8 \pm 4.12 \%)$ but not from the young $(25.59 \pm 2.86 \%)$ according to the analysis of the percentages of positive cells confirmed 


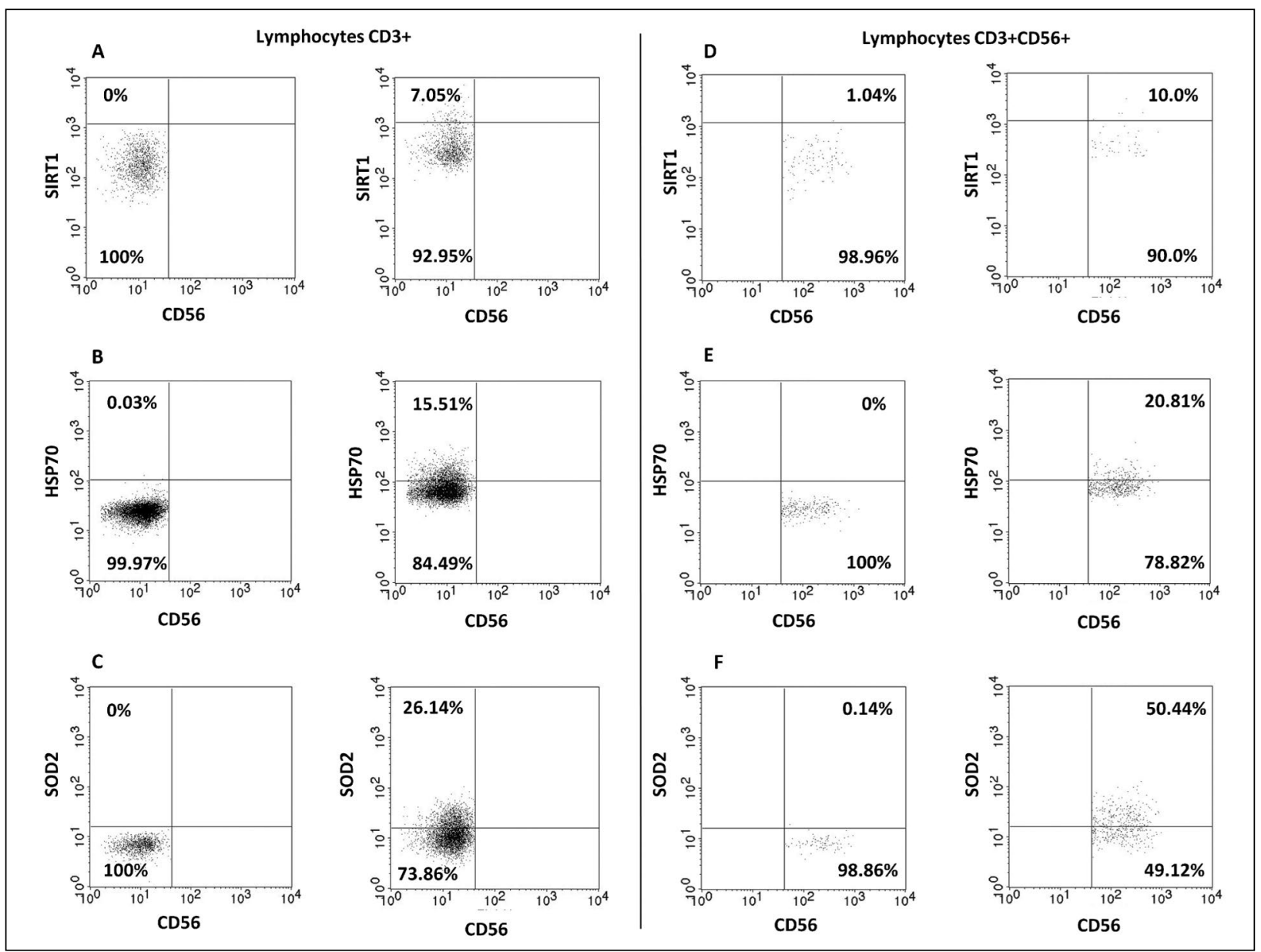

Figure 2. Flow cytometric analysis of $\mathrm{T}(\mathrm{CD} 3+)$ lymphocytes (left) and NKT-like (CD3+CD56+) cells (right). A. SIRT1 staining in T lymphocytes: left — isotype control; right — sample, T lymphocytes expressing SIRT1 were identified in the upper left quadrant. B. HSP70 staining in T lymphocytes: left — isotype control; right — sample, T lymphocytes expressing HSP70 were identified in the upper left quadrant. C. SOD2 staining in T lymphocytes: left — isotype control; right — sample, T lymphocytes expressing SOD2 were identified in the upper left quadrant. D. SIRT1 staining in NKT-like cells: left — isotype control; right - sample, NKT-like cells expressing SIRT1 were identified in the upper right quadrant. E. HSP70 staining in NKT-like cells: left — isotype control; right — sample, NKT-like cells expressing HSP70 were identified in the upper right quadrant. F. SOD2 staining in NKT-like cells: left — isotype control; right — sample, NKT-like cells expressing SOD2 were identified in the upper right quadrant.

also by the MFI analysis (Fig. 3E, F). Interestingly, in these analyses the expression of SOD2 in CD3+ cells of the young was significantly higher compared to $\mathrm{CD} 3+$ cells of the old (Fig. 3E, F). Similarly to HSP70, the expression of SOD2 was higher in NKT-like cells of the oldest $(68.30 \pm 6.7 \%)$ in comparison to the old $(36.01 \pm 4.51 \%)$ and the young $(43.45 \pm 3.06)$ in the analysis of the percentages of positive cells, confirmed also partly by the analysis of the relative expression (Fig. 3E, F). CD3+CD56+ cells appeared to express higher level of HSP70 in comparison to CD3 + cells when they were compared within the same age groups shown in the analysis of the relative expression and partly in the analysis of the percentages of positive cells (Fig. 3E, F).

\section{Correlations between expression levels} of the studied cellular protective proteins in $\mathrm{CD3}+$ and CD3+CD56+ cells

It was found that percentages of CD3+ cells expressing SIRT1 and HSP70 correlated positively with the age of participants, similarly to CD3+CD56+ cells which revealed additionally a positive correlation between the expression of SOD2 and the age. These correlations presented moderate values, with the exception of SOD2 in CD3 + CD56+ cells which revealed rather weak values (Table 2).

Some correlations between the expression of SIRT1 and other protective proteins were also observed in CD3+ and CD3+CD56+ cells. The percentage of SIRT1-expressing cells in these populations correlat- 


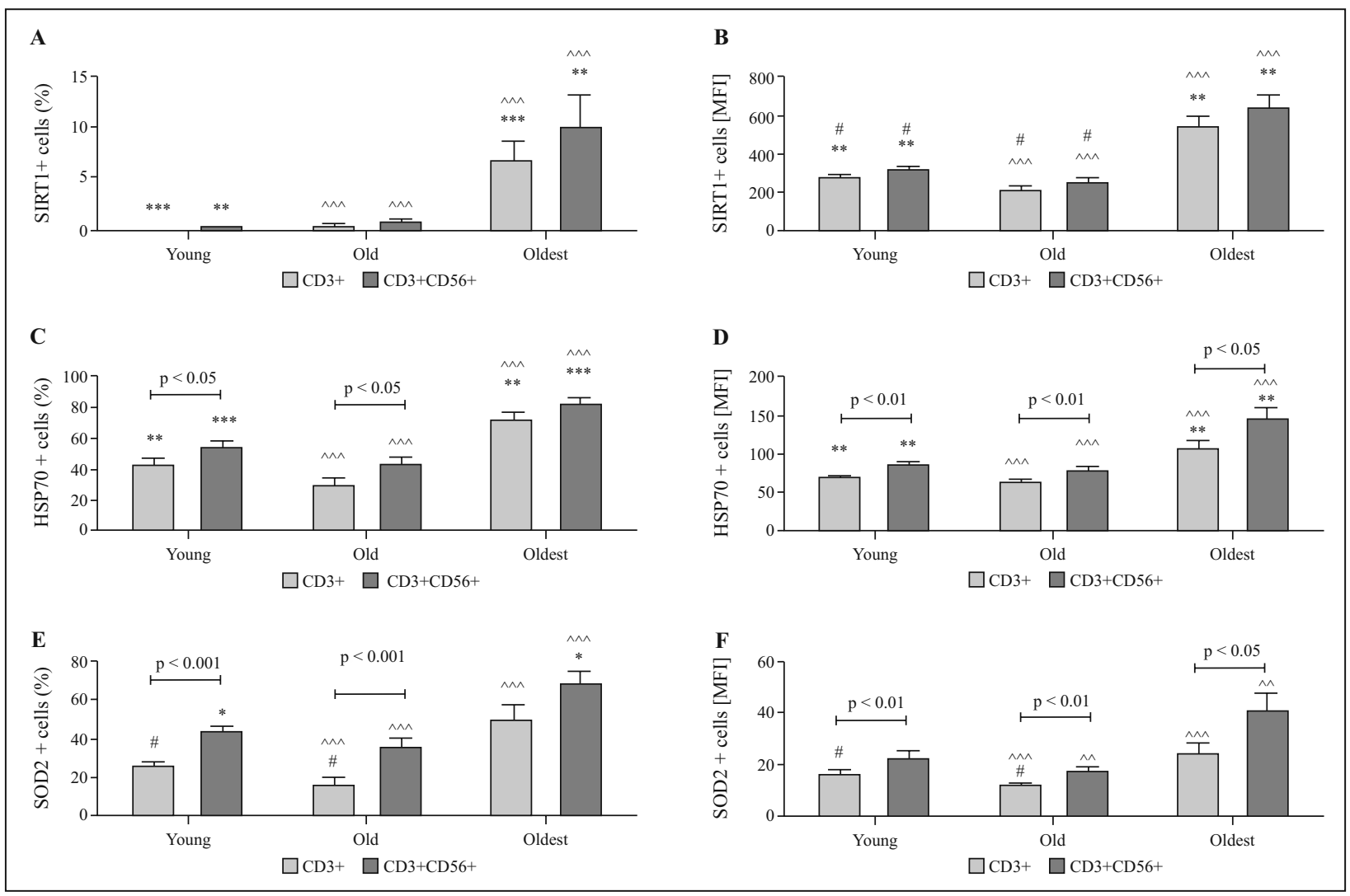

Figure 3. Expression of cellular protective proteins in $\mathrm{T}(\mathrm{CD} 3+)$ lymphocytes and NKT-like $(\mathrm{CD} 3+\mathrm{CD} 56+)$ cells of the young, old and the oldest. Data are presented as means \pm SEM and show expression of the studied protein in CD3+ and CD3 + CD56+ cells demonstrated as the percentage of cells with the expression of the particular protein (\%) and mean fluorescence intensity (MFI). The same symbols over bars $(\#, *, \wedge)$ denote statistically significant differences compared between: young $v$ s. old (marked with ${ }^{\#}$ ), young $v s$. oldest (marked with ${ }^{*}$ ) and old $v s$. oldest (marked with ${ }^{\wedge}$ ). 3 symbols denote $\mathrm{p}<0.001$; 2 symbols denote $\mathrm{p}<0.01 ; 1$ symbol denotes $\mathrm{p}<0.05$. Horizontal lines above paired bars denote statistically significant differences between CD3+ vs. CD3+CD56+ cells within the same age group. A. Expression of SIRT1 in CD3+ and CD3 + CD56+ cells (\%). B. Expression of SIRT1 in CD3+ and CD3+CD56+ cells (MFI). C. Expression of HSP70 in CD3+ and CD3+CD56+ cells (\%). D. Expression of HSP70 in CD3+ and CD3+CD56+ cells (MFI). E. Expression of SOD2 in $\mathrm{CD} 3+$ and $\mathrm{CD} 3+\mathrm{CD} 56+$ cells $(\%)$. F. Expression of SOD2 in CD3+ and CD3+CD56+ cells (MFI).

ed positively with the percentage of the relevant cells showing expression of: (i) HSP70 (high correlation) and (ii) SOD2 (moderate correlation). Similarly, the percentage of SOD2-expressing cells in CD3+ and $\mathrm{CD} 3+\mathrm{CD} 56+$ populations correlated positively with the percentage of the relevant cells showing expression of HSP70 (high correlation) (Table 2).

\section{Discussion}

NKT-like cells are "non-classical" NKT cells which represent highly differentiated $\mathrm{T}$ lymphocytes coexpressing several NK associated receptors (NKRs). Their number increases with age; however their contribution to the process of ageing is not entirely understood. Similarly, the expression of SIRT1, SOD2 and HSP70 in these cells concerning different age groups has yet not been investigated, to the best of our knowledge. The results of our study showing the positive correlation between the percentage of $\mathrm{CD} 3+$ and CD3+CD56+ lymphocytes with SIRT1 expression and the age of study participants suggest that increased expression of SIRT1 may be associated with human longevity. Our previous studies showed the similar relationships between the age and SIRT1 expression in NK cells as well as their subpopulations, i.e. CD56dim and CD56bright cells [20]. Sirtuin 1 was earlier described to affect lifespan and stress resistance in yeast [39, 40], invertebrates (e.g. Caenorhabditis elegans, Drosophila melanogaster) $[33,41,42]$ and mice [28]. We found that the expression of SIRT1 was the highest in the oldest seniors both in CD3+ and CD3+CD56+ cells and seemed to be higher in NKT-like cells, although the differences were not sta- 
Table 2. Correlation analysis of the study population

\begin{tabular}{|l|c|c|c|c|c|c|}
\hline \multirow{2}{*}{ Parameter } & \multicolumn{3}{|c|}{ T cells (CD3+) } & \multicolumn{3}{c|}{ NKT-like cells (CD3+CD56+) } \\
\cline { 2 - 7 } & SIRT1 & HSP70 & SOD2 & SIRT1 & HSP70 & SOD2 \\
\hline HSP70 & 0.690 & - & - & 0.694 & - & - \\
\hline SOD2 & 0.481 & 0.500 & - & 0.497 & 0.536 & - \\
\hline Age & 0.498 & 0.316 & ns & 0.378 & 0.372 & 0.253 \\
\hline
\end{tabular}

All values are presented as statistically significant Spearman's correlation coefficients (R). 'ns' denotes statistically not significant. Abbreviations: HSP — heat shock protein; SIRT — sirtuin; SOD — superoxide dismutase.

tistically significant both in the analysis of percentages of positive cells and MFI. Interestingly, the highest expression of SIRT1 in NKT-like cells of seniors over 85 corresponded to the highest expression of this protein in NK cells and their subsets, i.e. CD56dim and CD56bright cells of the oldest observed in our earlier studies [20]. The increase in the expression of SIRT1 mRNA in ageing CD4+ T lymphocytes was found also in mice [36].

Similarly to SIRT1, the highest expression of HSP70 was observed in both T and NKT-like lymphocytes of the oldest. However, in contrast to SIRT1, NKT-like cells expressed significantly more HSP70 compared to $\mathrm{T}$ cells in all age groups according to the analysis of the relative expression, confirmed mostly by the analysis of the percentages of positive cells. The expression of HSP70 correlated also positively with age. It is of interest that HSP70 expression in NKT-like cells was comparable with the expression of this protective protein in NK cells, including CD56dim and CD56bright subsets, or was even slightly higher in CD3 + CD56 + cells of all age groups. These findings were confirmed by both types of analysis, i.e. percentages of positive cells and MFI [20]. Our findings corresponded also to the results of the study performed on non-stimulated monocytes and lymphocytes. Njemini et al. reported higher levels of HSP70, HSP32 and HSP90, but not HSP27, assessed by flow cytometry in the studied cells of the elderly compared to young subjects. Thus, the increased expression of HSP70, and some other HSP proteins, seemed to be a general feature of NK cells and other leukocytes in ageing [34]. Interestingly, the subsequent studies performed by this group on $\mathrm{CD} 4+/ \mathrm{CD} 8+$ subsets of T lymphocytes and $\gamma \delta$, NK and B cells, revealed that the basal HSP70 levels in the various subpopulations of lymphocytes were comparable between the young and elderly subjects. However, higher expression of HSP70 was observed in CD45RO+ compared to CD45RA+ cells in both CD4+ and CD8+ subpopulations of T lymphocytes. According to Njemini et al., the ageing-related changes in basal HSP70 expression levels in PBL (peripheral blood lymphocyte) sub- populations were linked to the increased frequency of these lymphocyte subsets (i.e. memory $v$ s. naive phenotypes) than the elevated expression of these protective proteins in the cells [35].

Analogously to HSP70, the highest expression of SOD2 was observed in CD3+ and CD3+CD56+ cells of the oldest. NKT-like cells expressed significantly more SOD2 compared to $T$ cells in all age groups according to the analysis of the relative expression, confirmed in the group of the young and the old also by the analysis of the percentages of positive cells. However, the expression of SOD2 correlated positively with age only in NTK-like cells. It is interesting that similarly to HSP70, the expression of SOD2 in $\mathrm{CD} 3+\mathrm{CD} 56+$ cells of all age groups was comparable with the expression of this protein in NK cells and their subsets observed in our earlier studies [20]. However, contrary to HSP70, it was slightly lower in NKT-like cells compared to NK cells [20].

The high positive correlation between the expression of SIRT1 and HSP70 indicates that these proteins may interact in the maintenance of homeostasis in the process of immunosenescence. SIRT1 controls HSF1 (heat shock factor 1) activity, a transcription factor involved in the regulation of the expression of chaperons HSP70 and HSP90 activated in response to heat shock, hypoxia, misfolded proteins, free radicals and ATP depletion [43-45]. SIRT1 was found also to activate several transcription factors of the FOXO family that promote the expression of stress response genes including SOD2 [33]. This relationship was observed also in the presented study as a moderate positive correlation between the expression of SIRT1 and SOD2. Manganese superoxide dismutase similarly to sirtuin 1 seems to play a role in longevity, at least in some species, i.e. Drosophila [46] and mice [47]. We also found a positive correlation between the age and SOD2 expression, observed, however, only in $\mathrm{CD} 3+\mathrm{CD} 56+$ cells.

The analysis of the number and percentage of $\mathrm{CD} 3+$ and $\mathrm{CD} 3+\mathrm{CD} 56+$ lymphocytes performed in our study corresponded to the investigations of 
the other groups and showed the increase in the number of NKT-like cells in the process of ageing [4, $6,48]$ and decrease in the number and percentage of T lymphocytes [48-50].

The results of our studies provided some new findings concerning the expression of cellular protective proteins SIRT1, HSP70 and SOD2 in CD3+ and $\mathrm{CD} 3+\mathrm{CD} 56+$ cells regarding their contribution to longevity. They complete our earlier studies concerning participation of NK cells and their subsets in the process of immunosenescence [20, 21, 51]. These studies may provide also a good base for some subsequent, more detailed investigations concerning the expression of cellular protective proteins in T lymphocytes and their subsets, including NKT-like cells.

\section{Conclusions}

Our study shows the increased expression of cellular protective proteins involved in stress response, i.e. SIRT1, HSP70 and SOD2, in both T lymphocytes and NKT-like cells of the oldest seniors. Their expression correlates with the age of participants and this phenomenon seems to correspond to longevity. Interestingly, significantly higher expression of cellular protective proteins HSP70 and SOD2 (and the similar tendency in SIRT1 expression) was found in NKT-like cells (CD3+CD56+) in comparison to T $(\mathrm{CD} 3+)$ lymphocytes in the all studied age groups. This phenomenon may underline the role of NKT-like cells in providing a link between innate and adaptive immunity in ageing process.

\section{Acknowledgements}

This study was supported by grant N N404 597640 from the National Science Centre (NCN, Poland) and by ST-12 internal funds of the Medical University of Gdansk, Poland.

\section{References}

1. Tarazona R, DelaRosa O, Alonso C, et al. Increased expression of NK cell markers on $\mathrm{T}$ lymphocytes in aging and chronic activation of the immune system reflects the accumulation of effector/senescent T cells. Mechanisms of Ageing and Development. 2001; 121(1-3): 77-88, doi: 10.1016/ s0047-6374(00)00199-8.

2. Pita-López ML, Pera A, Solana R. Adaptive Memory of Human NK-like CD8 T-Cells to Aging, and Viral and Tumor Antigens. Front Immunol. 2016; 7: 616, doi: 10.3389/fimmu.2016.00616, indexed in Pubmed: 28066426.

3. Torina A, Guggino G, La Manna MP, et al. The Janus Face of NKT Cell Function in Autoimmunity and Infectious Diseases. Int J Mol Sci. 2018; 19(2), doi: 10.3390/ijms19020440, indexed in Pubmed: 29389901.

4. Peralbo E, Alonso C, Solana R. Invariant NKT and NKT-like lymphocytes: two different $\mathrm{T}$ cell subsets that are differentially affected by ageing. Exp Gerontol. 2007; 42(8): 703-708, doi: 10.1016/j.exger.2007.05.002, indexed in Pubmed: 17604928.

5. Panda A, Arjona A, Sapey E, et al. Human innate immunosenescence: causes and consequences for immunity in old age. Trends Immunol. 2009; 30(7): 325-333, doi: 10.1016/j. it.2009.05.004, indexed in Pubmed: 19541535.

6. Solana R, Mariani E. NK and NK/T cells in human senescence. Vaccine. 2000; 18(16): 1613-1620, doi: 10.1016/s0264410x(99)00495-8.

7. Lemster BH, Michel JJ, Montag DT, et al. Induction of CD56 and TCR-Independent Activation of T Cells with Aging. The Journal of Immunology. 2008; 180(3): 1979-1990, doi: 10.4049/jimmunol.180.3.1979.

8. Godfrey DI, MacDonald HR, Kronenberg M, et al. NKT cells: what's in a name? Nat Rev Immunol. 2004; 4(3): 231-237, doi: 10.1038/nri1309, indexed in Pubmed: 15039760.

9. Jiang Y, Cui X, Cui C, et al. The function of CD3+CD56+ NKT-like cells in HIV-infected individuals. Biomed Res Int. 2014; 2014: 863625, doi: 10.1155/2014/863625, indexed in Pubmed: 24779018.

10. Kelly-Rogers J, Madrigal-Estebas L, O'Connor T, et al. Activation-induced expression of CD56 by T cells is associated with a reprogramming of cytolytic activity and cytokine secretion profile in vitro. Hum Immunol. 2006; 67(11): 863-873, doi: 10.1016/j.humimm.2006.08.292, indexed in Pubmed: 17145366 .

11. Dunne J, Lynch S, O'Farrelly C, et al. Selective Expansion and Partial Activation of Human NK Cells and NK Receptor-Positive T Cells by IL-2 and IL-15. The Journal of Immunology. 2001; 167(6): 3129-3138, doi: 10.4049/jimmunol.167.6.3129.

12. Chen YJ, Liao HF. NK/NKT Cells and Aging. International Journal of Gerontology. 2007; 1(2): 65-76, doi: 10.1016/ s1873-9598(08)70025-5.

13. Franceschi C, Bonafé M, Valensin S, et al. Inflamm-aging: An Evolutionary Perspective on Immunosenescence. Annals of the New York Academy of Sciences. 2006; 908(1): 244-254, doi: 10.1111/j.1749-6632.2000.tb06651.x.

14. Licastro F, Candore G, Lio D, et al. Innate immunity and inflammation in ageing: a key for understanding age-related diseases. Immun Ageing. 2005; 2: 8, doi: 10.1186/17424933-2-8.

15. Müller L, Pawelec G. As we age: Does slippage of quality control in the immune system lead to collateral damage? Ageing Res Rev. 2015; 23(Pt A): 116-123, doi: 10.1016/j. arr.2015.01.005, indexed in Pubmed: 25676139.

16. Frasca D, Blomberg BB. Inflammaging decreases adaptive and innate immune responses in mice and humans. Biogerontology. 2016; 17(1): 7-19, doi: 10.1007/s10522-015-9578-8, indexed in Pubmed: 25921609.

17. Bektas A, Schurman SH, Sen R, et al. Aging, inflammation and the environment. Exp Gerontol. 2018; 105: 10-18, doi: 10.1016/j.exger.2017.12.015, indexed in Pubmed: 29275161.

18. Höhn A, Weber D, Jung T, et al. Happily (n)ever after: Aging in the context of oxidative stress, proteostasis loss and cellular senescence. Redox Biol. 2017; 11: 482-501, doi: 10.1016/j.redox.2016.12.001, indexed in Pubmed: 28086196.

19. Kaszubowska L, Kaczor JJ, Hak L, et al. Sensitivity of natural killer cells to activation in the process of ageing is related to the oxidative and inflammatory status of the elderly. J Physiol Pharmacol. 2011; 62(1): 101-109, indexed in Pubmed: 21451215 . 
20. Kaszubowska L, Foerster J, Kaczor JJ, et al. Expression of cellular protective proteins SIRT1, HSP70 and SOD2 correlates with age and is significantly higher in NK cells of the oldest seniors. Immun Ageing. 2017; 14: 3, doi: 10.1186/s12979-0170085-4, indexed in Pubmed: 28127381.

21. Kaszubowska L, Foerster J, Kaczor JJ, et al. NK cells of the oldest seniors represent constant and resistant to stimulation high expression of cellular protective proteins SIRT1 and HSP70. Immun Ageing. 2018; 15: 12, doi: 10.1186/s12979018-0115-x, indexed in Pubmed: 29541147.

22. Afolayan AJ, Teng RJ, Eis A, et al. Inducible HSP70 regulates superoxide dismutase- 2 and mitochondrial oxidative stress in the endothelial cells from developing lungs. Am J Physiol Lung Cell Mol Physiol. 2014; 306(4): L351-L360, doi: 10.1152/ajplung.00264.2013, indexed in Pubmed: 24375796.

23. Zemanovic S, Ivanov MV, Ivanova LV, et al. Dynamic Phosphorylation of the C Terminus of Hsp70 Regulates the Mitochondrial Import of SOD2 and Redox Balance. Cell Rep. 2018; 25(9): 2605-2616.e7, doi: 10.1016/j.celrep.2018.11.015, indexed in Pubmed: 30485823.

24. Martínez de Toda I, De la Fuente M. The role of Hsp70 in oxi-inflamm-aging and its use as a potential biomarker of lifespan. Biogerontology. 2015; 16(6): 709-721, doi: 10.1007/ s10522-015-9607-7, indexed in Pubmed: 26386684.

25. Santos L, Escande C, Denicola A. Potential Modulation of Sirtuins by Oxidative Stress. Oxid Med Cell Longev. 2016; 2016: 9831825, doi: 10.1155/2016/9831825, indexed in Pubmed: 26788256.

26. Langley E. Human SIR 2 deacetylates p53 and antagonizes PML/p53-induced cellular senescence. The EMBO Journal. 2002; 21(10): 2383-2396, doi: 10.1093/emboj/21.10.2383.

27. Vaziri H, Dessain S, Eaton E, et al. hSIR2SIRT1 Functions as an NAD-Dependent p53 Deacetylase. Cell. 2001; 107(2): 149-159, doi: 10.1016/s0092-8674(01)00527-x.

28. Brunet A, Sweeney LB, Sturgill JF, et al. Stress-dependent regulation of FOXO transcription factors by the SIRT1 deacetylase. Science. 2004; 303(5666): 2011-2015, doi: 10.1126/science.1094637, indexed in Pubmed: 14976264.

29. Motta M, Divecha N, Lemieux M, et al. Mammalian SIRT1 Represses Forkhead Transcription Factors. Cell. 2004; 116(4): 551-563, doi: 10.1016/s0092-8674(04)00126-6.

30. Yeung F, Hoberg JE, Ramsey CS, et al. Modulation of NF-kappaB-dependent transcription and cell survival by the SIRT1 deacetylase. EMBO J. 2004; 23(12): 2369-2380, doi: 10.1038/sj.emboj.7600244, indexed in Pubmed: 15152190.

31. Longo VD. Linking sirtuins, IGF-I signaling, and starvation. Exp Gerontol. 2009; 44(1-2): 70-74, doi: 10.1016/j.exger.2008.06.005, indexed in Pubmed: 18638538.

32. van der Horst A, Tertoolen LGJ, de Vries-Smits LMM, et al. FOXO4 is acetylated upon peroxide stress and deacetylated by the longevity protein hSir2(SIRT1). J Biol Chem. 2004; 279(28): 28873-28879, doi: 10.1074/jbc.M401138200, indexed in Pubmed: 15126506.

33. Merksamer PI, Liu Y, He W, et al. The sirtuins, oxidative stress and aging: an emerging link. Aging (Albany NY). 2013; 5(3): 144-150, doi: 10.18632/aging.100544, indexed in Pubmed: 23474711

34. Njemini R, Bautmans I, Lambert M, et al. Heat shock proteins and chemokine/cytokine secretion profile in ageing and inflammation. Mech Ageing Dev. 2007; 128(7-8): 450-454, doi: 10.1016/j.mad.2007.06.003, indexed in Pubmed: 17644159 .

35. Njemini R, Demanet C, Mets T. Aging-related differences in basal heat shock protein 70 levels in lymphocytes are linked to altered frequencies of lymphocyte subsets. Aging Cell. 2008; 7(4): 498-505, doi: 10.1111/j.1474-9726.2008.00401.x, indexed in Pubmed: 18489731.

36. Li Y, Ohms SJ, Sun C, et al. NF- $\kappa$ B controls Il2 and Csf 2 expression during $\mathrm{T}$ cell development and activation process. Mol Biol Rep. 2013; 40(2): 1685-1692, doi: 10.1007/s11033012-2219-2, indexed in Pubmed: 23079711.

37. Folstein M, Folstein S, McHugh P. "Mini-mental state". Journal of Psychiatric Research. 1975; 12(3): 189-198, doi: 10.1016/0022-3956(75)90026-6.

38. Katz S. Studies of Illness in the Aged. JAMA. 1963; 185(12): 914, doi: 10.1001/jama.1963.03060120024016.

39. Kaeberlein M, McVey M, Guarente L. The SIR2/3/4 complex and SIR2 alone promote longevity in Saccharomyces cerevisiae by two different mechanisms. Genes \& Development. 1999; 13(19): 2570-2580, doi: 10.1101/gad.13.19.2570.

40. Lin SJ. Requirement of NAD and SIR2 for Life-Span Extension by Calorie Restriction in Saccharomyces cerevisiae. Science. 2000; 289(5487): 2126-2128, doi: 10.1126/science.289.5487.2126.

41. Tissenbaum HA, Guarente L. Increased dosage of a sir-2 gene extends lifespan in Caenorhabditis elegans. Nature. 2001; 410(6825): 227-230, doi: 10.1038/35065638, indexed in Pubmed: 11242085.

42. Rogina B, Helfand SL. Sir2 mediates longevity in the fly through a pathway related to calorie restriction. Proc Natl Acad Sci U S A. 2004; 101(45): 15998-16003, doi: 10.1073/ pnas.0404184101, indexed in Pubmed: 15520384.

43. Westerheide SD, Anckar J, Stevens SM, et al. Stress-inducible regulation of heat shock factor 1 by the deacetylase SIRT1. Science. 2009; 323(5917): 1063-1066, doi: 10.1126/ science.1165946, indexed in Pubmed: 19229036.

44. Beier UH, Akimova T, Liu Y, et al. Histone/protein deacetylases control Foxp3 expression and the heat shock response of T-regulatory cells. Curr Opin Immunol. 2011; 23(5): 670-678, doi: 10.1016/j.coi.2011.07.002, indexed in Pubmed: 21798734.

45. West JD, Wang Y, Morano KA. Small molecule activators of the heat shock response: chemical properties, molecular targets, and therapeutic promise. Chem Res Toxicol. 2012; 25(10): 2036-2053, doi: 10.1021/tx300264x, indexed in Pubmed: 22799889.

46. Paul A, Belton A, Nag S, et al. Reduced mitochondrial SOD displays mortality characteristics reminiscent of natural aging. Mech Ageing Dev. 2007; 128(11-12): 706-716, doi: 10.1016/j. mad.2007.10.013, indexed in Pubmed: 18078670.

47. Rodriguez-Iturbe B, Sepassi L, Quiroz Y, et al. Association of mitochondrial SOD deficiency with salt-sensitive hypertension and accelerated renal senescence. J Appl Physiol (1985). 2007; 102(1): 255-260, doi: 10.1152/japplphysiol.00513.2006, indexed in Pubmed: 17023572.

48. Miyaji $\mathrm{C}$, Watanabe $\mathrm{H}$, Minagawa $\mathrm{M}$, et al. Numerical and functional characteristics of lymphocyte subsets in centenarians. J. Clin. Immunol. 1997; 17: 420-429.

49. Wei Li, Zhao J, Wu W, et al. Decreased absolute numbers of CD3 T cells and CD8 T cells during aging in herpes zoster patients. Sci Rep. 2017; 7(1): 15039, doi: 10.1038/s41598-01715390-w, indexed in Pubmed: 29118328.

50. Tavares SM, Junior Wd, Lopes E Silva MR. Normal lymphocyte immunophenotype in an elderly population. Rev Bras Hematol Hemoter. 2014; 36(3): 180-183, doi: 10.1016/j.bjhh.2014.03.021, indexed in Pubmed: 25031056 
51. Kaszubowska L, Foerster J, Schetz D, et al. CD56 bright cells respond to stimulation until very advanced age revealing increased expression of cellular protective proteins
SIRT1, HSP70 and SOD2. Immun Ageing. 2018; 15: 31, doi: 10.1186/s12979-018-0136-5, indexed in Pubmed: 30534181 .

Submitted: 19 December, 2018 Accepted after reviews: 19 December, 2018 Available as AoP: 2 January, 2019 